\title{
Screening of Dirac flavor structure in the seesaw and neutrino mixing
}

\author{
Manfred Lindner ${ }^{1(a)}$, Michael A. Schmidt ${ }^{2(a)}$, and Alexei Yu. Smirnov ${ }^{3(a),(b),(c)}$ \\ (a) Physik-Department T30, Technische Universität München, \\ James-Franck-Straße, 85748 Garching, Germany \\ (b) The Abdus Salam International Centre for Theoretical Physics, I-34100 Trieste, Italy \\ (c) Institute for Nuclear Research, Russian Academy of Science, Moscow, Russia
}

\begin{abstract}
We consider the mechanism of screening of the Dirac flavor structure in the context of the double seesaw mechanism. As a consequence of screening, the structure of the light neutrino mass matrix, $m_{\nu}$, is determined essentially by the structure of the (Majorana) mass matrix, $M_{S}$, of new super-heavy (Planck scale) neutral fermions $S$. We calculate effects of the renormalization group running in order to investigate the stability of the screening mechanism with respect to radiative corrections. We find that screening is stable in the supersymmetric case, whereas in the standard model it is unstable for certain structures of $M_{S}$. The screening mechanism allows us to reconcile the (approximate) quark-lepton symmetry and the strong difference of the mixing patterns in the quark and lepton sectors. It opens new possibilities to explain a quasi-degenerate neutrino mass spectrum, special "neutrino" symmetries and quark-lepton complementarity. Screening can emerge from certain flavor symmetries or Grand Unification.
\end{abstract}

\footnotetext{
${ }^{1}$ E-mail: lindner@ph.tum.de

${ }^{2}$ E-mail: mschmidt@ph.tum.de

${ }^{3}$ E-mail: smirnov@ictp.trieste.it
} 


\section{Introduction}

Quarks and leptons show an apparent correspondence which suggests their common origin [1]. The observation is based on the pattern of quantum numbers and the fact that both quarks and leptons come in three fermionic families (generations). This allows quarks and leptons to be embedded into unique multiplets associated with extended gauge symmetries [1, 2, 3, 4. In spite of lack of further experimental confirmations, the quark-lepton symmetry and unification [1, 2, 3, 4, are still the most appealing concepts in physics beyond the Standard Model (SM).

Tiny neutrino masses emerge naturally from the seesaw mechanism [5], 6], which implies the existence of right handed neutrino fields $\nu_{R}$ and new high energy scale. The seesaw scale turns out to be numerically rather close to the scale of Grand Unification (GU), which emerges from the unification of gauge couplings. It seems as if both gauge and fermion unification point in the same direction of certain Grand Unified Theory, like SO(10) GUTs [3].

On the basis of quark-lepton unification (symmetry) one would expect similarities between the mass and mixing patterns of quarks and leptons. However, observations [7, 8] do not support this expectation. Indeed,

- Neutrino masses and mixings differ strongly from those in the quark sector. The hierarchy of neutrino masses, if exists, is weaker than the quark mass hierarchy. The 2-3 leptonic mixing is maximal or close to maximal, whereas the corresponding quark mixing is very small. The 1-2 leptonic mixing is large but not maximal and it seems smaller than the 2-3 leptonic mixing. In contrast, the 1-2 quark mixing - the largest quark mixing - is small. The only common feature is that the 1-3 mixings are small in both sectors.

- Data seem to indicate a particular "neutrino symmetry" 9] which does not show up in other sectors of the theory. This includes maximal or nearly maximal 2-3 mixing and relatively small 1-3 mixing. If the neutrino mass spectrum would turn out to be quasi-degenerate $^{1}$, this could imply certain symmetry too. However, it is very difficult to extend the suggested symmetries not only onto the quarks but also to charged leptons and models (e.g. [1]) which realize such neutrino symmetries are quite involved.

- In the context of the seesaw mechanism, the observed values of neutrino masses require the masses of the right-handed (RH) neutrinos to be $M_{N} \lesssim 10^{14} \mathrm{GeV}$, i.e. two orders of magnitude below the Grand Unification scale, $M_{G U}$. Furthermore, the data implies a certain flavor structure and a hierarchy of the RH neutrino mass matrix which may indicate that the scale of $\mathrm{RH}$ neutrino masses emerges as a combination of the GU-scale and some other scale close to the Planck mass $M_{P l}$ :

$$
M_{N} \sim \frac{M_{G U}^{2}}{M_{P l}}
$$

- The experimental values of the quark and lepton mixing angles between the first and second generations appear to sum up to the maximal mixing angle:

$$
\theta_{12}+\theta_{C} \approx \frac{\pi}{4}
$$

\footnotetext{
${ }^{1}$ This would be necessary if the evidence for neutrino-less double beta decay [10] would be confirmed.
} 
with the leptonic mixing angle $\theta_{12}$ and the Cabibbo angle $\theta_{C}$. The interpretation of such a quark-lepton complementarity relation [12, 13, 14, 15, is rather controversial. If it is not a numerical accident, relation (2) implies some quark-lepton connection which is not present in ordinary GUTs. It may require some additional structure in the leptonic sector which produces maximal mixing and therefore has certain symmetry.

All these observations can be considered as hints against a simple unification of quarks and leptons.

There exist various attempts to reconcile the possible quark-lepton symmetry (unification) and the strong difference of patterns of masses and mixing in two sectors. The main problem is that the strong hierarchy of eigenvalues and small mixings of the quark mass matrices imply a certain hierarchy in the Dirac type Yukawa couplings which propagates due to the symmetry/unification to the lepton sector (see, however [16]). One possible solution is that dominant contribution to the neutrino masses, e.g., from the Yukawa coupling with a Higgs triplet, has no analogy in the quark sector. However, even in this case, it seems rather unnatural that some Yukawa interactions have a particular symmetry which is not realized for other Yukawa interactions. In the context of seesaw mechanism the observed features of neutrino masses and mixing can be related to a particular structure of the Majorana mass matrix of the RH neutrinos [17, 18].

In this paper in order to reconcile the observed pattern of the neutrino masses and mixings and quark-lepton symmetry, we elaborate on the double seesaw mechanism [19. Specifically, we will discuss a version where the flavor structures of the Dirac type Yukawa couplings cancel completely [17, 20, in the light neutrino mass matrix. The properties of the neutrino mass matrix are entirely determined by physics (in particular, symmetries) at energies above the Grand unification scale. We will call this "screening of the Dirac flavor structure" or simply "screening" and we will show it may lead to a natural solution of the above mentioned problems.

The paper is organized as follows. In sec. 2 we describe the screening mechanism. We consider the renormalization group effects and the stability of the mechanism with respect to radiative corrections in sec. 3. Various applications of the mechanism are studied in sec. 4. In particular, we consider a possibility of the degenerate neutrino mass spectrum, existence of particular neutrino symmetries, and the quark-lepton complementarity. In sec. 5 we discuss how the screening mechanism can be realized in GUT and models with family symmetries. Conclusions are presented in sec. [6.

\section{The flavor screening mechanism}

In addition to the SM particles we introduce the three right-handed neutrinos, $\nu_{i R} \equiv N_{i}^{c}$, $(i=1,3,3)$, three left-handed singlets $S_{i}$ and Higgs boson singlet $\sigma$. The leptonic interactions are assumed to have the following features:

1. The SM leptonic doublets $L_{i}$ have Yukawa couplings $Y_{\nu}$ with $N \equiv\left(\nu_{R}\right)^{c}$;

2. The Majorana mass terms of the right-handed neutrinos, $N$ are forbidden or strongly suppressed;

3. The right-handed neutrinos $N_{i}$ have Yukawa couplings $Y_{N}$ with singlets $S_{i}$ and a Higgs boson $\sigma$; 
4. The singlets $S_{i}$ have Majorana mass terms, thus violating the lepton number.

Under these assumptions the following Yukawa interactions and mass terms appear in the Lagrangian

$$
-\mathscr{L}=L^{T} Y_{\nu} N \phi+S^{T} Y_{N} N \sigma+\frac{1}{2} S^{T} M_{S} S+L^{T} Y_{e} l^{c} \phi_{d}+\text { h.c. },
$$

(the flavor indices are omitted here). The last term in eq. 3] is the Yukawa coupling which generates masses for the charge leptons; in the Standard model: $\phi_{d}=\tilde{\phi} \equiv i \sigma_{2} \phi^{*}$. After the Higgs bosons develop the non-zero VEVs, $\langle\phi\rangle$ and $\langle\sigma\rangle$, the neutrino mass matrix is generated in the basis $(\nu, N, S)$

$$
\mathcal{M}=\left(\begin{array}{ccc}
0 & Y_{\nu}\langle\phi\rangle & 0 \\
Y_{\nu}^{T}\langle\phi\rangle & 0 & Y_{N}^{T}\langle\sigma\rangle \\
0 & Y_{N}\langle\sigma\rangle & M_{S}
\end{array}\right)
$$

For $\langle\phi\rangle \ll\langle\sigma\rangle \ll M_{S}$ it realizes the double (or cascade) seesaw mechanism [19]. (The case of singular matrices $M_{S}$ will be considered separately in sec. 4.5). Block diagonalization of the matrix (44) leads to the mass matrix of light neutrinos

$$
m_{\nu}^{0}=\left[\frac{\langle\phi\rangle}{\langle\sigma\rangle}\right]^{2} Y_{\nu}\left(Y_{N}\right)^{-1} M_{S}\left(Y_{N}^{T}\right)^{-1} Y_{\nu}^{T}
$$

This matrix has the remarkable feature that the Dirac type Yukawa coupling matrices cancel provided that $Y_{\nu}$ and $Y_{N}$ are proportional, i.e., if

$$
Y_{\nu}=c \cdot Y_{N}
$$

with constant $c$ being typically of the order unity. (Its deviation from unity can be e.g. due to the renormalization group effects.) As a result of the cancellation the mass matrix of light neutrinos becomes

$$
m_{\nu}=c^{2}\left[\frac{\langle\phi\rangle}{\langle\sigma\rangle}\right]^{2} M_{S},
$$

where the neutrino mixings emerge entirely from the flavor structure in $M_{S}$ [17. The flavor structure of the Dirac type Yukawa couplings is completely eliminated, while it still determines all the features of the quark masses and mixings. We will call such a cancellation the screening of the Dirac flavor structure in the lepton mixing or briefly, screening. Screening is a consequence of the double seesaw (4) and the proportionality (6). These features can emerge from certain flavor symmetries or Grand Unification, as it will be discussed in sec. 5 . The scale of neutrino masses is determined by the scales of the double seesaw. Taking

$$
\langle\phi\rangle=v_{E W}, \quad\langle\sigma\rangle \sim M_{G U} \sim 10^{16} \mathrm{GeV}, \quad M_{S} \sim M_{P l}
$$

one finds from (7)

$$
m_{\nu} \sim\left[\frac{v_{E W}}{M_{G U}}\right]^{2} M_{P l},
$$

which lies in the phenomenologically required range, while the flavor structure of the neutrino mass matrix, and consequently the mass ratios and mixings are determined by the Planck scale physics ${ }^{2}$.

\footnotetext{
${ }^{2}$ In the paper [21] the double seesaw matrix (4) has been considered with only one new singlet $S$ (so that the $Y_{N}$ is the 3 component column). However, such a matrix can not reproduce the required mass spectrum of
} 
The Majorana mass matrix of the right handed neutrinos, $M_{N}$, generated after the first seesaw equals

$$
M_{N}=-\langle\sigma\rangle^{2} Y_{N}^{T} M_{S}^{-1} Y_{N}
$$

For the mass scales given in eq. (8) we obtain $M_{N} \sim M_{G U}^{2} / M_{P l} \leq 10^{14} \mathrm{GeV}$ which reproduce the relation (11) required by phenomenology.

An attractive scenario could be that some symmetry in the sector of singlets $S$ exists. This symmetry leads to a particular and simple structure of $M_{S}$ at high scales. The symmetry is broken at lower scales and incomplete screening, if exists, can be related to this breaking. In fact, phenomenology may require deviations from complete screening. Perturbations might e.g. explain the observed mass splitting of the light neutrinos in the case of a degenerate spectrum of the singlets $S_{f}$. In this connection one can explore the following origins of perturbations (breaking) of the structure (46):

- Nonzero elements in the 1-1, 1-3 and 2-2 blocks of matrix in eq. (44): These blocks have different gauge properties and the origin of these perturbations can be quite different.

- Mismatch between $Y_{\nu}$ and $Y_{N}$ destroying cancellation.

- Perturbations in $M_{S}$. They may follow from the Planck scale physics. If the eigenvalues of $M_{S}$ have some hierarchy then one needs to take into account the renormalization effects due to the Yukawa interactions of $S, N$ and $\sigma$ which influence the structure of matrix $M_{S}$.

A structure of the mass matrix for $(\nu, N, S)$ with non-zero 1-3 block (the direct $\nu S$-terms) has been considered in ref. 22. Under certain conditions the matrix leads to the linear dependence (instead of quadratic or no dependence in our case) of the light neutrino mass matrix on the Dirac flavor structure. In a sense this realizes the half-screening effect.

Notice that one can introduce the Majorana mass matrix of the $\mathrm{RH}$ neutrinos in the form (10) immediately without invoking the double seesaw mechanism. This is done in [23] where form $M_{N}=-\langle\sigma\rangle^{2} Y_{N}^{T} Y_{N}+\delta M_{N}$ with $\delta M_{N}$ being the correction matrix has been postulated. In contrast, we propose a model for the structure (10) based on the double seesaw mechanism and additional flavor symmetry or/and Grand Unification.

\section{Screening and renormalization group effect}

In this section we will consider effects of the radiative corrections [24, 25, 26, 27] on the screening mechanism. The proportionality relation (6) required for screening, holds presumably at the large scale of $M_{S}$ or above that. However, the couplings $Y_{N}$ and $Y_{\nu}$ have different gauge properties and their renormalization group running to the EW scale leads to different radiative corrections which may destroy the screening effect. On the other hand, small mismatch of the renormalized Yukawa matrices (partial screening) may explain some of the observed properties of the neutrino mass spectrum and mixings.

light neutrinos for any structure of $Y_{N}$. The complete mass spectrum consists of three Majorana neutrinos with masses (in our notations) $\sim M_{S}, \sim\langle\sigma\rangle^{2} / M_{S}, \sim\langle\phi\rangle^{2} M_{S} /\langle\sigma\rangle^{2}$, and two pseudo-Dirac neutrinos with masses at the electroweak scale: $\sim\langle\phi\rangle$. There is only one light neutrino. A possibility to get a correct spectrum in the model [21] is to introduce the direct non-negligible contribution to the RH neutrino masses (2-2 block) of the form $Y_{\nu}^{T} M^{\prime} Y_{\nu}$, where $M^{\prime}$ is some $3 \times 3$ matrix to be tuned to fit the data. 


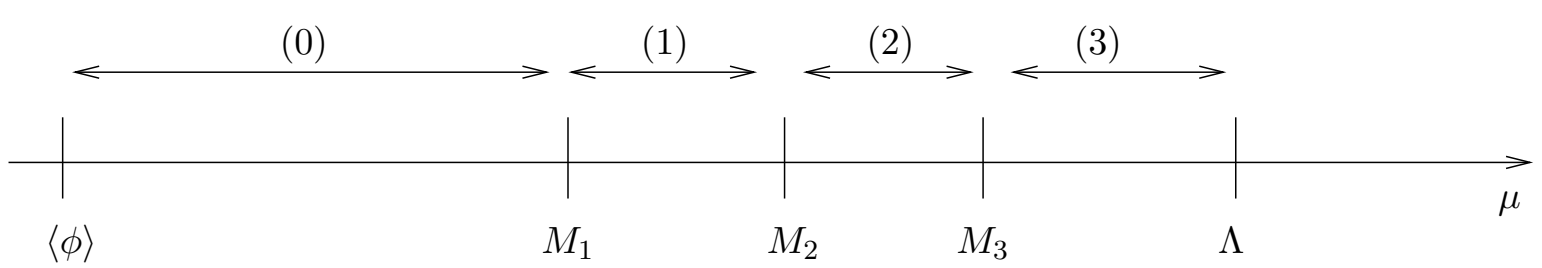

Figure 1: The thresholds due to masses of the right-handed neutrinos and the intervals of the RG running.

In what follows we will consider a minimal scenario - corrections due to the SM and MSSM interactions. Presence of other physics beyond the standard model can lead to additional renormalization effects.

\subsection{Renormalization group effects}

Here we will consider the renormalization group effects below a certain scale $\Lambda$ which in turn is somehow below the masses of singlets $M_{S}$ :

$$
M_{i} \ll \Lambda \leq M_{S},
$$

here $M_{i}$ are the masses of RH neutrinos (see Fig. 1). We consider $M_{S}$ as the mass matrix of singlets at $\Lambda$, so that possible renormalization effects on $M_{S}$ due to the couplings $Y_{N}$ are included.

Let us stress that for the hierarchical Yukawa matrix $Y_{\nu}$, and consequently $Y_{N}$, the mass spectrum of the RH neutrinos is (in general) strongly hierarchical: $M_{N} \sim Y_{\nu}^{2}$. Therefore effects of the RG running between different mass thresholds is crucial [28, 29, 30, 23, 31.

Let us introduce the effective operator $\stackrel{(n)}{M}_{M}$ which generates neutrino masses in the basis $(\nu, N)$

$$
\mathscr{L}=-\left(\nu^{T}, N^{T}\right) \stackrel{(n)}{O_{M}}\left(\nu^{T}, N^{T}\right)^{T} .
$$

The superscript $(n)$ designates the number of right-handed neutrinos which are not decoupled at a given energy scale, that is, neutrinos in the effective theory. This superscript will denote also a range of RG-running with a given number of $\mathrm{RH}$ neutrinos, and we use also the notation $\stackrel{(n-m)}{Z}=(\stackrel{(n)}{Z}(n+1) \quad(m-1)(m)$

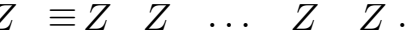

Below the scale $\Lambda$ the singlets $S$ are integrated out, and the effective operator $\stackrel{(3)}{O}_{M}$ can be written as

$$
\stackrel{(3)}{O_{M}}(\Lambda)=\left(\begin{array}{cc} 
& (3) \\
0 & Y_{\nu} \phi \\
(3) & (3) \\
Y_{\nu}^{T} \phi & M_{N}
\end{array}\right)
$$

Here

$$
\stackrel{(3)}{M_{N}}=-\langle\sigma\rangle^{2} \stackrel{(3)}{Y_{N}^{T}} M_{S}^{-1} \stackrel{(3)}{Y_{N}}
$$

is the mass matrix of the three $\mathrm{RH}$ neutrinos at the scale $\Lambda$, and $Y_{\nu}$ is the matrix of Yukawa couplings at $\Lambda$. 


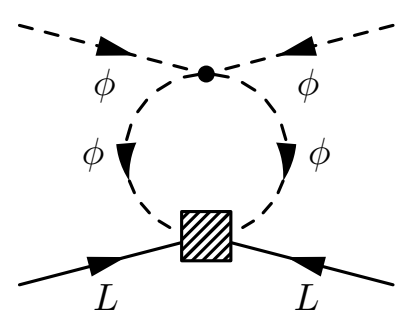

(a) Higgs self-coupling

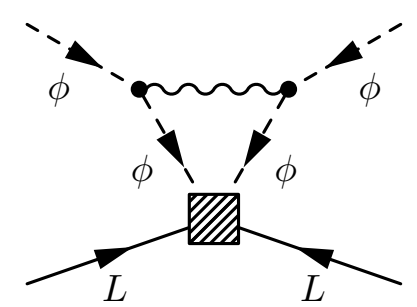

(b) Gauge interactions

Figure 2: The $\mathrm{d}=5$ operator vertex corrections. Shown are additional divergent diagrams in the effective theory.

The effect of the RG evolution can be split in effects coming from the renormalization of the wave functions and the vertex corrections. It turns out, that the RG corrections can be factorized in the leading log (LL) approximation. So, in general, the renormalizations of $Y_{\nu}$, $M_{N}$ and $m_{\nu} \phi^{2} /\langle\phi\rangle^{2}$ (operator of the light neutrino masses) are given by

$$
\begin{gathered}
Y_{\nu} \stackrel{\mathrm{RG}}{\longrightarrow} Z_{\text {ext }}^{T} Y_{\nu} Z_{N} \\
M_{N} \stackrel{\mathrm{RG}}{\longrightarrow} Z_{N}^{T} M_{N} Z_{N} \\
m_{\nu} \phi^{2} /\langle\phi\rangle^{2} \stackrel{\mathrm{RG}}{\longrightarrow} Z_{\text {ext }}^{T} m_{\nu} \phi^{2} /\langle\phi\rangle^{2} Z_{\text {ext }} Z_{\kappa} .
\end{gathered}
$$

Here $Z_{\text {ext }}$ combines the renormalization effect of the left-handed doublets $L$, the Higgs doublet $\phi$, and the vertex correction to $Y_{\nu} ; Z_{N}$ denotes the wave function renormalization effect of the $\mathrm{RH}$ neutrinos $N$. In order to simplify the presentation, we define the wave function renormalization so that the usual powers of $1 / 2$ factors are absent. Eq. (17) describes renormalization of the effective dimension $d=5$ operator which appears after decoupling (integration out) of the corresponding RH neutrino. Apart from renormalization of the wave functions and vertices which exist in the SM model this operator has additional vertex corrections given by the diagrams in Fig. 2] The RG effect due to these diagrams denoted by $\stackrel{(n)}{Z}_{\kappa}$ plays a crucial role in the discussion of the stability of screening These $d=5$ operator corrections are absent in the supersymmetric version of theory due to the non-renormalization theorem [32, 33.

We describe the RG effects in the effective theory, where the heavy $\mathrm{RH}$ neutrinos are decoupled successively ${ }^{3}$ [28, 29, 30, 23, 31] as depicted in Fig. 1. In each step (interval between mass thresholds) we first calculate the RG correction to the matrices. We diagonalize the resulting matrices at the lower end of the interval, i.e., at $\mu=M_{i}$ and then decouple $N_{i}$, $(i=3,2,1)$. We will denote the renormalization factors in the leading log approximation $(n) \quad(n)$ by $Z=1+\delta Z$. This notation is also used for parameters of the effective theory. The renormalization factors in the extended (by RH neutrinos) SM and the MSSM are given in appendix $\mathrm{A}$

Let us describe the main steps of the renormalization procedure, which is especially simple in the basis where matrix $Y_{\nu}$ is diagonal. (This basis, however, does not coincide with the flavor basis.)

\footnotetext{
${ }^{3}$ The running between mass thresholds of $\mathrm{RH}$ neutrinos has been treated analytically in the approximation of strongly hierarchical and diagonal Yukawa matrix in ref. 23]. Here we present a general consideration required for our approach.
} 
1). The RG evolution between $\Lambda$ and $M_{3}$ yields the operator $O_{M}^{(3)}$ at $M_{3}$

$$
\stackrel{(3)}{O_{M}}\left(M_{3}\right)=\left(\begin{array}{cc}
0 & (3) \\
0 & Z_{\text {ext }}^{T} Y_{\nu} \phi \stackrel{(3)}{Z_{N}} \\
& (3)(3)(3) \\
\ldots & Z_{N}^{T} M_{N} Z_{N}
\end{array}\right)
$$

Performing a rotation of the $\mathrm{RH}$ neutrinos $N=\stackrel{(3)}{U_{N}} N^{\prime}$ we reduce the renormalized RH neutrino mass matrix to the form

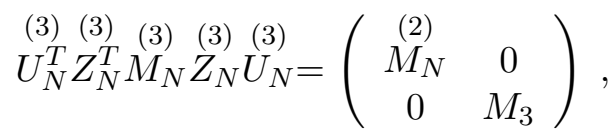

where $\stackrel{(2)}{M_{N}}$ is $2 \times 2$ (in general off-diagonal) mass matrix. Let us split the $3 \times 3$ Dirac type Yukawa coupling matrix in (18) after this rotation into two parts as

$$
\stackrel{(3)}{Z_{\mathrm{ext}}^{T}} \stackrel{(3)}{Y_{\nu}} \stackrel{(3)}{Z}_{N} \stackrel{(3)}{U_{N}} \equiv\left(\begin{array}{l}
(2) \\
Y_{\nu},
\end{array}, y_{3}\right)
$$

where $y_{3}$ is the 3 component column of the Yukawa couplings of $\nu$ and $N_{3}$, and $\stackrel{(2)}{\nu}_{\nu}$ is the rest $3 \times 2$ sub-matrix. Then in the rotated basis the operator (18) can be written as

$$
\stackrel{(3)}{O_{M}}\left(M_{3}\right)=\left(\begin{array}{ccc}
0 & \stackrel{(2)}{Y_{\nu} \phi} & y_{3} \phi \\
& \stackrel{(2)}{M_{N}} & 0 \\
\cdots & 0 & M_{3}
\end{array}\right)
$$

Below the scale $M_{3}$ the neutrino $N_{3}$ is integrated out and from (21) we obtain

$$
\stackrel{(2)}{O_{M}}\left(M_{3}\right)=\left(\begin{array}{cc}
-y_{3} M_{3}^{-1} y_{3}^{T} \phi^{2} & \stackrel{(2)}{Y}_{\nu} \\
& (2) \\
\cdots & M_{N}
\end{array}\right) .
$$

Notice that the $d=5$ operator is formed in 1-1 block due to decoupling of $N_{3}$.

2). Let us consider the RG running in the interval $M_{2}-M_{3}$. Similarly to the first step we can write the operator $O_{M}$ at the scale $M_{2}$ (threshold of $N_{2}$ ) as

$$
\stackrel{(2)}{O_{M}}\left(M_{2}\right)=\left(\begin{array}{cc}
-Z_{\mathrm{ext}}^{T} \stackrel{(2)}{Z_{\kappa}} y_{3} M_{3}^{-1} y_{3}^{T} \phi^{2} \stackrel{(2)}{Z_{\mathrm{ext}}} & Z_{\mathrm{ext}}^{T} \stackrel{(2)}{Y}_{\nu} \stackrel{(2)}{Z_{N}} \\
\ldots & Z_{N}^{T} \stackrel{(2)}{M} \mathscr{( 2 )}_{N}
\end{array}\right)
$$

(2)

Here we have included the corrections $Z_{\kappa}$ to the $\mathrm{d}=5$ operator.

By applying the rotation $N^{\prime}=\stackrel{(2)}{U_{N}} N^{\prime \prime}$ the renormalized mass matrix of the RH neutrinos is diagonalized:

$$
\stackrel{(2)}{U_{N}^{T}} \stackrel{(2)}{T}_{N}^{T} \stackrel{(2)}{M} \stackrel{(2)}{Z}_{N} \stackrel{(2)}{U}_{N} \equiv\left(\begin{array}{cc}
(1) & \\
M_{N} & 0 \\
0 & M_{2}
\end{array}\right)
$$


The renormalized Yukawa matrix is then split as

$$
\stackrel{(2)}{Z_{\text {ext }}^{T}} \stackrel{(2)}{Y_{\nu}} \stackrel{(2)}{Z}_{N} \stackrel{(2)}{U_{N}} \equiv\left(\begin{array}{ll}
(1) & \\
Y_{\nu}, & y_{2}
\end{array}\right)
$$

where $\stackrel{(1)}{Y}_{\nu}$ and $y_{2}$ are two component columns. Decoupling the second neutrino $N_{2}$ we obtain

$$
\stackrel{(1)}{O_{M}}\left(M_{2}\right)=\left(\begin{array}{cc}
-\stackrel{(2)}{Z}_{\mathrm{ext}}^{T} \stackrel{(2)}{K}_{\kappa} y_{3} M_{3}^{-1} y_{3}^{T} \phi^{2} \stackrel{(2)}{Z}_{\mathrm{ext}}-y_{2} M_{2}^{-1} y_{2}^{T} \phi^{2} & \stackrel{(1)}{Y_{\nu}} \phi \\
\ldots & \stackrel{(1)}{M_{N}}
\end{array}\right)
$$

3). Running the matrix down to the lowest see-saw scale $M_{1}$ and integration out $N_{1}$ yields

$$
\begin{aligned}
\stackrel{(0)}{O_{M}}\left(M_{1}\right)= & -\stackrel{(1-2)}{Z_{\text {ext }}^{T}} \stackrel{(1-2)}{Z_{\kappa}} y_{3} M_{3}^{-1} y_{3}^{T} \phi^{2} \stackrel{(1-2)}{Z_{\text {ext }}} \\
& \stackrel{(1)}{(1)}\left[\stackrel{(1)}{Z_{\kappa}} y_{2} M_{2}^{-1} y_{2}^{T}+\stackrel{(1)}{Y_{\nu}} M_{N}^{-1} \stackrel{(1)}{Y}_{\nu}^{T}\right] \phi^{2} \stackrel{(1)}{Z_{\mathrm{ext}}}
\end{aligned}
$$

4). Finally, evolving $O_{M}^{(0)}\left(M_{1}\right)$ down to the EW scale, we obtain (after $\phi$ develops a VEV) the mass matrix of light neutrinos

$$
m_{\nu}=-\langle\phi\rangle^{2} \stackrel{(0-3)}{Z_{\text {ext }}^{T}} \stackrel{(3)}{Y_{\nu} Z_{N} U_{N}^{(3)}\left({ }^{(3)}\right.}\left(\begin{array}{cc}
K_{12} & 0 \\
& \frac{(0-2)}{Z_{\kappa}} \\
0 & \frac{(3)}{M_{3}}
\end{array}\right) \stackrel{(3)}{U_{N}^{T} Z_{N}^{T} Y_{\nu}^{T} Z_{\text {ext }}^{(3)}},
$$

with

$$
K_{12} \equiv \stackrel{(2)}{Z_{N}} \stackrel{(2)}{U}_{N}\left(\begin{array}{cc}
\stackrel{(0)}{Z_{\kappa}} & 0 \\
\stackrel{(1)}{M} & 0 \\
M_{N} & (0-1) \\
0 & \frac{\left(Z_{\kappa}\right.}{M_{2}}
\end{array}\right) \stackrel{(2)^{T}}{U_{N}} \stackrel{(2)}{T}_{N}^{T}
$$

This expression can be presented in a simpler and transparent way. Using definitions of matrices $\stackrel{(2)}{U_{N}}$ and $\stackrel{(3)}{U_{N}}$ in eqs. (24, 19) we can rewrite $m_{\nu}$ as

$$
m_{\nu}=-\langle\phi\rangle^{2} Z_{\text {ext }}^{T}\left[\begin{array}{l}
(3) \\
Y_{\nu}
\end{array} X_{N} M_{N}^{-1} Y_{\nu}^{T}\right] Z_{\text {ext }}
$$

where

$$
X_{N} \equiv \stackrel{(3)}{Z_{N}} \stackrel{(3)}{U_{N}} \stackrel{(2)}{Z_{N}^{\prime}} \stackrel{(2)}{U_{N}^{\prime}} Z_{\kappa} \stackrel{(2)}{U_{N}^{\prime \dagger}} Z_{N}^{\prime} Z_{N}^{\prime-1} \stackrel{(3)}{U_{N}^{\dagger}} \stackrel{(3)}{Z_{N}^{-1}}
$$

describes the RG effects due to the running between the thresholds. Here

$$
\stackrel{(2)}{Z_{N}^{\prime}} \equiv\left(\begin{array}{cc}
(2) & \\
Z_{N} & 0 \\
0 & 1
\end{array}\right), \quad \stackrel{(2)}{U_{N}^{\prime}} \equiv\left(\begin{array}{cc}
(2) & \\
U_{N} & 0 \\
0 & 1
\end{array}\right)
$$




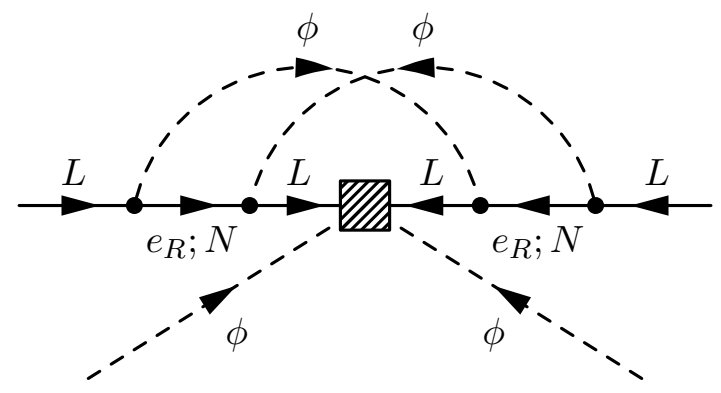

Figure 3: Examples of the two loop diagram which destroy factorization of the vertex corrections to the effective neutrino mass matrix.

and

$$
Z_{\kappa} \equiv \operatorname{diag}\left(\begin{array}{ccc}
(0) & (0-1) & (0-2) \\
Z_{\kappa}, & Z_{\kappa}, & Z_{\kappa}
\end{array}\right)
$$

is the matrix of effective $d=5$ operator corrections (fig. 21). The expressions for $Z_{\text {ext }}$ are given in appendices A.1 and A.2 Formulas (28) and (30) for $m_{\nu}$ are our main results which we will analyze in the following sections.

\subsection{Stability of screening}

Inserting the expression for the matrix $M_{N}$, eq. (14), into eq. (30) we obtain

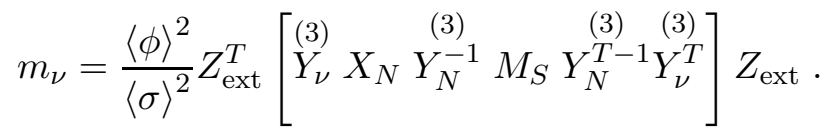

If the equality (6) is satisfied, the neutrino mass matrix becomes

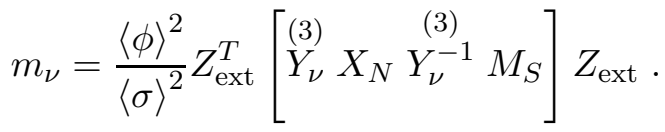

Therefore screening is reproduced and the dependence of $m_{\nu}$ on the Yukawa (Dirac) couplings disappears if $X_{N}=I$. The expression (35) coincides with that in (7) up to external renormalization. In turn, according to eq. (31) the equality $X_{N}=I$ holds provided that $Z_{\kappa}=I$, that is, when the $d=5$ operator corrections are absent. This is automatically satisfied in the supersymmetric theory, but the corrections are present in the SM and its non-supersymmetric extensions.

Note that the $\mathrm{d}=5$ operator corrections are due to the gauge interactions and self interactions of the Higgs boson, which are by themselves flavor blind. However, they influence the flavor structure of the light neutrino mass matrix due to difference of masses of the $\mathrm{RH}$ neutrinos and therefore different threshold effects.

\subsection{Beyond leading log approximation}

Beyond the leading log (LL) approximation the RG contributions still factorize except for the $\mathrm{d}=5$ operator corrections. Thus, our analysis does hold beyond LL in the MSSM, but it does not apply in the SM due to diagrams like in Fig. 3. However, the 2 loop contributions to the 
external renormalization are smaller than the 1 loop corrections by a factor of $y^{2} / 16 \pi^{2} \leq 0.01$ and the 1 loop threshold corrections are not enhanced by large logarithms.

For certain structures of $M_{S}$ (see sec. (4) the additional 2 loop diagrams lead to corrections to the renormalization of the effective neutrino mass operator which could be comparable to the 1 loop corrections. However, assuming the same hierarchy in the neutrino Yukawa couplings as in the up-type quark Yukawa couplings, these contribution are further suppressed since the heaviest right-handed neutrino is already integrated out. Therefore all 2 loop contributions in the effective theory are suppressed by $y_{2}^{2} / 16 \pi^{2} \leq 10^{-6}$ compared to the 1 loop contributions. Altogether higher loop contributions are less than $10 \%$ of the 1 loop corrections and will be neglected in the following.

\subsection{Effects of vertex corrections}

In order to study the $\mathrm{d}=5$ operator corrections in the (non-supersymmetric) standard model in more details we introduce the matrix $V_{N}$ which diagonalizes the $\mathrm{RH}$ neutrino mass matrix at $\Lambda$ :

$$
V_{N}^{T} \stackrel{(3)}{M} V_{N}=\langle\sigma\rangle^{2} V_{N}^{T} \stackrel{(3)}{Y_{N}^{T}} M_{S}^{-1} \stackrel{(3)}{Y_{N}} V_{N}=D_{N} \equiv \operatorname{diag}\left(M_{1}, M_{2}, M_{3}\right) .
$$

In the lowest order approximation: $\stackrel{(2)}{Z}_{N}=\stackrel{(3)}{Z}_{N}=1$, and according to (19) and (24) we obtain

$$
V_{N}=\stackrel{(3)}{U_{N}} \stackrel{(2)}{U_{N}^{\prime}} \cdot
$$

Therefore the matrix $X_{N}$ (31) can be rewritten in the form

$$
X_{N}=V_{N} Z_{\kappa} V_{N}^{\dagger}=I+V_{N} \delta Z_{\kappa} V_{N}^{\dagger},
$$

where

$$
\delta Z_{\kappa} \equiv Z_{\kappa}-I .
$$

Plugging this expression for $X_{N}$ in (34) we find

$$
m_{\nu} \equiv \frac{\langle\phi\rangle^{2}}{\langle\sigma\rangle^{2}} Z_{\mathrm{ext}}^{T}\left[I+\stackrel{(3)}{Y_{\nu}} V_{N} \delta Z_{\kappa} V_{N}^{\dagger} \stackrel{(3)}{Y}_{\nu}^{-1}\right] M_{S} Z_{\mathrm{ext}}
$$

Finally, using (36) we can rewrite $m_{\nu}$ as

$$
m_{\nu} \equiv\left\langle\phi^{2}\right\rangle Z_{\mathrm{ext}}^{T}\left[\frac{1}{\langle\sigma\rangle^{2}} M_{S}+\stackrel{(3)}{Y_{\nu}} V_{N}\left(\delta Z_{\kappa} D_{N}^{-1}\right) V_{N}^{T} \stackrel{(3)}{Y_{\nu}^{T}}\right] Z_{\mathrm{ext}} .
$$

According to this expression the effects of $d=5$ operator corrections are reduced to renormalization of masses of the $\mathrm{RH}$ neutrinos since $\delta Z_{\kappa}$ is diagonal.

\section{Applications of the Dirac structure screening}

Here we apply the results obtained in the previous section to several phenomenologically interesting structures of $M_{S}$. We study effects of the radiative corrections on the light neutrino 
mass matrix. The matrix $M_{S}$ will be defined in the basis where the equality of the Yukawa couplings ([6) is fulfilled.

In the previous sections we have found $m_{\nu}$ in the basis where the neutrino Yukawa coupling matrix $Y_{\nu}$ is diagonal. Now we will discuss $m_{\nu}^{f}$ - the neutrino mass matrix in the flavor basis where the charge lepton mass matrix $Y_{e}$ is diagonal. It is related to $m_{\nu}$ as

$$
m_{\nu}^{f}=U_{e}^{T} m_{\nu} U_{e},
$$

where $U_{e}$ is the transformation of left handed charged lepton components which diagonalizes the matrix $Y_{e}$ at the electroweak scale. The radiative corrections to $Y_{e}$ are in general small due to the strong mass hierarchy.

\subsection{Quasi-degenerate neutrino spectrum}

Let us first consider $M_{S}$ which is proportional to the unit matrix at $\Lambda$, i.e.,

$$
M_{S}=M_{S}^{0} I
$$

This choice is apparently basis independent and we can take therefore $Y_{\nu}=Y_{N}=\operatorname{diag}\left(y_{1}, y_{2}, y_{3}\right)$. The right-handed neutrino mass matrix is diagonal and strongly hierarchical:

$$
M_{N}=-Y_{N}^{T} M_{S}^{-1} Y_{N}\langle\sigma\rangle^{2}=\frac{\langle\sigma\rangle^{2}}{M_{S}} \operatorname{diag}\left(y_{1}^{2}, y_{2}^{2}, y_{3}^{2}\right)
$$

Therefore $V_{N}=I$ and we find

$$
m_{\nu}^{f} \equiv \frac{\langle\phi\rangle^{2}}{\langle\sigma\rangle^{2}} M_{S}^{0} U_{e}^{T} Z_{\text {ext }}^{T}\left[I+\delta Z_{\kappa}\right] Z_{\text {ext }} U_{e} .
$$

The corrections are also diagonal ${ }^{4}$

$$
\delta Z_{\kappa}=\left[\exp \left(\mathcal{A} \operatorname{diag}\left(0, \ln \frac{y_{2}^{2}}{y_{3}^{2}}, \ln \frac{y_{1}^{2}}{y_{3}^{2}}\right)\right)-I\right] \sim \mathcal{O}(0.1)
$$

where

$$
\mathcal{A} \equiv \frac{1}{16 \pi^{2}}\left(\lambda+\frac{9}{10} g_{1}^{2}+\frac{3}{2} g_{2}^{2}\right) .
$$

This leads to splittings of the light neutrino masses which would be degenerate otherwise.

Note that the external corrections (due to the wave function renormalization of the lefthanded leptons, eq. (A.1), and the vertex corrections to the neutrino Yukawa couplings, eq. (A.1) ), are described in general by off-diagonal matrices due to the mismatch of $Y_{e}$ and $Y_{\nu}$ structures. As the charged lepton Yukawa couplings are also strongly hierarchical, the largest flavor dependent correction is the one to the 3-3 element. Neglecting the off-diagonal entries, it can be estimated as

$$
-2 \frac{y_{\tau}^{2}}{16 \pi^{2}} \ln \frac{\langle\phi\rangle}{\Lambda}-4 \frac{y_{3}^{2}}{16 \pi^{2}} \ln \frac{M_{3}}{\Lambda} \sim \mathcal{O}(0.1)
$$

where the second term (due to the neutrino Yukawa coupling) dominates. It has the same order of magnitude as the correction due to the $d=5$ operator renormalization in eq. (45).

\footnotetext{
${ }^{4}$ We assume a strong hierarchy in $Y_{\nu}$ and use $Y_{\nu} \sim Y_{u}$ for the numerical estimates.
} 
Let us now comment on a possibility to explain the neutrino data. In the non-supersymmetric version the mass split, $\Delta m$, generated by the $\mathrm{d}=5$ operator corrections, $\Delta m=m_{0} \delta Z$, leads to $\Delta m^{2}=2 m_{0} \Delta m=2 m_{0}^{2} \delta Z=(2-8) \cdot 10^{-3} \mathrm{eV}^{2}$ for the overall scale $m_{0}=(0.1-0.2) \mathrm{eV}$. This can reproduce the atmospheric mass split, but it is too large for the solar mass split. The ratio of solar and atmospheric mass squared differences,

$$
\frac{\Delta m_{21}^{2}}{\Delta m_{32}^{2}} \approx \frac{m_{2}-m_{1}}{m_{3}-m_{2}} \sim \mathcal{O}(1)
$$

does not fit the observations. The external corrections do not improve the situation either. Therefore some other (non-radiative) contribution is required to compensate the 1-2 mass split. Mixings can also be generated by small (non-radiative) corrections.

In the supersymmetric version we have $\delta Z_{\kappa}=0$ and $Z_{Y_{\nu}}=I$, so that the mass splitting is produced by the external renormalization only:

$$
m_{\nu}^{f}=\frac{\langle\phi\rangle^{2}}{\langle\sigma\rangle^{2}} M_{S}^{0} U_{e}^{T} Z_{\mathrm{ext}}^{T} U_{e} Z_{\mathrm{ext}}
$$

In the flavor basis we obtain the mass split due to Yukawa couplings coming from the external renormalization:

$$
\exp \left[-\frac{1}{8 \pi^{2}} \operatorname{diag}\left(y_{e}^{2}, y_{\mu}^{2}, y_{\tau}^{2}\right) \ln \frac{\langle\phi\rangle}{\Lambda}\right],
$$

where the neutrino Yukawa couplings are neglected. This can provide the atmospheric mass split and the mixings should be generated again by correction to the zero order structure.

Next, we consider for $M_{S}$ the "triangle" structure

$$
M_{S}=M_{S}^{0}\left(\begin{array}{ccc}
1 & 0 & 0 \\
0 & 0 & 1 \\
0 & 1 & 0
\end{array}\right)
$$

in the basis where the neutrino Yukawa matrices are diagonal. In lowest order it produces a degenerate mass spectrum and maximal 2-3 mixing of the light neutrinos. This matrix leads to a spectrum of RH neutrinos with two heavy degenerate states and one relatively light state:

$$
M_{1 N}=\frac{\langle\sigma\rangle^{2} y_{1}^{2}}{M_{S}^{0}}, \quad M_{2 N}=-M_{3 N}=\frac{\langle\sigma\rangle^{2} y_{2} y_{3}}{M_{S}^{0}} .
$$

The renormalization interval "(2)" (see fig. 1) is therefore absent and the matrix of $\mathrm{d}=5$ operator corrections can be written as

$$
\delta Z_{\kappa}=\left[\exp \left(\delta \stackrel{(1)}{Z_{\kappa}}\right)-1\right] \operatorname{diag}(0,1,1), \quad \delta \stackrel{(1)}{Z_{\kappa}}=\mathcal{A} \ln \frac{y_{1}^{2}}{y_{2} y_{3}}
$$

where $\mathcal{A}$ is defined in eq. (46). The state $N_{1}$ decouples and maximal mixing is realized in the 2-3 block of $V_{N}$. Using this feature and eq. (53) we find from eq. (40)

$$
m_{\nu}^{f} \equiv Z_{\mathrm{ext}}^{T} \frac{\langle\phi\rangle^{2}}{\langle\sigma\rangle^{2}} M_{S}^{0} U_{e}^{T} \stackrel{(0)}{Z_{\kappa}}\left(\begin{array}{ccc}
1 & 0 & 0 \\
0 & 0 & 1-\delta \stackrel{(1)}{Z}_{\kappa}^{(1)} \\
0 & 1-\delta \stackrel{Z_{\kappa}}{ } & 0
\end{array}\right) Z_{\mathrm{ext}} U_{e}
$$


Therefore the $\mathrm{d}=5$ operator corrections do not destroy the triangular structure, but they lead to mass splits between the degenerate pair and the isolated state:

$$
\frac{\Delta m}{m}=\delta \stackrel{(1)}{Z}_{\kappa}
$$

In the supersymmetric version $\delta \stackrel{(1)}{Z}_{\kappa}=0$, so that the original "triangle" structure is renormalized by the external corrections only. In this case, one also needs perturbations of the original screening structure in order to get the correct mixings and mass split.

As a third possibility we consider for $M_{S}$ the "triangle" structure which leads to a degenerate spectrum and maximal 1-2 mixing:

$$
M_{S}=M_{S}^{0}\left(\begin{array}{lll}
0 & 1 & 0 \\
1 & 0 & 0 \\
0 & 0 & 1
\end{array}\right)
$$

Similar considerations as above results in the mass spectrum of RH neutrinos with two light degenerate states and an isolated heavier state:

$$
M_{1 N}=-M_{2 N}=\frac{\langle\sigma\rangle^{2} y_{1} y_{2}}{M_{S}^{0}}, \quad M_{3 N}=\frac{\langle\sigma\rangle^{2} y_{3}^{2}}{M_{S}^{0}} .
$$

For the light neutrinos we find

$$
m_{\nu} \equiv Z_{\mathrm{ext}}^{T} \frac{\langle\phi\rangle^{2}}{\langle\sigma\rangle^{2}} M_{S}^{0} U_{e}^{T} \stackrel{(0)}{Z_{\kappa}}\left(\begin{array}{ccc}
0 & 1 & 0 \\
1 & 0 & 0 \\
0 & 0 & 1-\delta \stackrel{(2)}{Z}_{\kappa}
\end{array}\right) Z_{\mathrm{ext}} U_{e}
$$

where

$$
\delta \stackrel{(2)}{Z_{\kappa}}=\exp \left(\mathcal{A} \ln \frac{y_{1} y_{2}}{y_{3}^{2}}\right)-1 .
$$

The corrections due to running of the $\mathrm{d}=5$ operator are of the same order as in the previous case. The mass split

$$
\Delta m_{32}^{2}=2 m_{0} \Delta m_{32}=-2 m_{0}^{2} \delta \stackrel{(2)}{Z_{\kappa}}=(2-8) \cdot 10^{-3} \mathrm{eV}^{2},
$$

for $m_{0}=(0.08-0.16) \mathrm{eV}$ can explain the atmospheric neutrino data. The external renormalization contributes in the same way as for $M_{S} \propto I$.

In the supersymmetric version the "triangle" structure is preserved. Therefore the original matrix $M_{S}$ as given in eq. (56) should be perturbed in order to produce phenomenological acceptable mixings.

Let us comment on the possibility to generate the lepton asymmetry of the Universe via the CP-violating decay of the lightest right handed neutrino(s) 34. In the case of diagonal $M_{S}$ the mass of the lightest neutrino $N_{1}$ is in the TeV range. So, according to [35] the produced asymmetry is too small to explain the Baryon asymmetry via sphaleron effect. In contrast, in the case of a triangular matrix $M_{S}$ (58) there are two quasi-degenerate $\mathrm{RH}$ neutrinos with masses $M \simeq 10^{6} \mathrm{GeV}$, and resonant leptogenesis can produce the required asymmetry. 


\subsection{Perturbations of $M_{S}$}

Let us consider perturbations of the structure of $M_{S}$ (which can be required by phenomenology) and effect radiative corrections on these perturbed structures.

As an example we take the matrix

$$
M_{S}=M_{S}^{0}\left(\begin{array}{ccc}
1 & 0 & 0 \\
0 & x & 1 \\
0 & 1 & 0
\end{array}\right)
$$

with $x$ being a free parameter. Now the second and third neutrinos are no longer degenerate and the renormalization factor $\stackrel{(2)}{Z}_{\kappa}$ in the interval "(2)" between their masses appears. Approximating $\stackrel{(n)}{Z}_{\kappa}$ by $1+\mathcal{A} \ln \left(M_{n} / M_{n+1}\right)$ we obtain for the light neutrinos

$$
m_{\nu}^{f}=\frac{\langle\phi\rangle^{2}}{\langle\sigma\rangle^{2}} M_{S}^{0} U_{e}^{T} \stackrel{(0)}{Z}_{\kappa} Z_{\text {ext }}^{T}\left(\begin{array}{ccc}
1 & 0 & 0 \\
\ldots & x(1+\mathcal{A}) m_{22}^{\text {th }} & 1+\mathcal{A} m_{23}^{\text {th }} \\
\ldots & \ldots & \mathcal{A} m_{33}^{\text {th }}
\end{array}\right) Z_{\text {ext }} U_{e},
$$

where the threshold dependent corrections, $m_{i j}^{\text {th }}$, equal

$$
\begin{aligned}
& m_{22}^{\text {th }}=-3 \ln \lambda+\left(\frac{1}{2}-\frac{\lambda^{2}}{x^{2} y}\right) \ln \frac{y-1}{y+1}, \\
& m_{23}^{\text {th }}=-3 \ln \lambda+\frac{1}{2 y} \ln \frac{y-1}{y+1}, \\
& m_{33}^{\text {th }}=\frac{1}{x y} \ln \frac{y-1}{y+1} .
\end{aligned}
$$

Here $y \equiv \sqrt{1+4\left(\frac{\lambda}{x}\right)^{2}}$ and $\lambda \equiv y_{2} / y_{3}$. (The logarithms depend on the ratios on the $\mathrm{RH}$ neutrino masses $M_{2} / M_{3}$.)

The nonzero 3-3 element is generated in eq. (62) by the radiative corrections. Furthermore, this element can be enhanced by small parameter $x$ in the denominator, provided that $\lambda$ is also small enough. Indeed, from eq. (63) we find explicitly

$$
\left(m_{\nu}\right)_{33}=\left\{\begin{aligned}
\frac{2 \mathcal{A}}{x} \ln \frac{\lambda}{x}, & x \gg \lambda \\
-\frac{1.26 \mathcal{A}}{x}, & x=2 \lambda \\
-\frac{\mathcal{A} x}{2 \lambda^{2}}, & x \ll \lambda
\end{aligned}\right.
$$

Since $\mathcal{A} \sim 10^{-2}$, the $3-3$ element can be of the order 1 or even more if, e.g., $\lambda \ll x<10^{-2}$. Thus, a quasi-degenerate $M_{S}$ with nearly maximal 2-3 mixing leads after (non-supersymmetric) renormalization group corrections to the hierarchical mass matrix $m_{\nu}$ with small mixing. The texture (61) is not stable against quantum corrections, since the structure of $m_{\nu}$ strongly differs from the original structure of $M_{S}$.

This example shows that radiative corrections can substantially modify the original texture of $M_{S}$ in the light neutrino mass matrix for particular $M_{S}$. In other words, radiative corrections may destroy screening. 
Apparently the corrections are small if $\lambda \ll x \sim 1$. This corresponds to phenomenologically important case of the dominant $2-3$ block:

$$
M_{S}=M_{S}^{0}\left(\begin{array}{ccc}
\epsilon & 0 & 0 \\
0 & x & 1 \\
0 & 1 & x
\end{array}\right)
$$

with $x \sim 1$ and $\epsilon \ll 1$.

In the supersymmetric version of model screening is stable, since there are no $d=5$ operator corrections due to non-renormalization theorem. Note also that exact off-diagonal structures of $M_{S}$ are stable, but small perturbations are unstable with respect to radiative corrections.

\subsection{Neutrino symmetry}

The mass matrix $M_{S}$ of the singlets $S$ has no analogy in the quark sector, and general, it is not related to the quark mass matrices. Furthermore, $M_{S}$ is generated at a higher scale, than the GUT scale. Therefore it is possible that $M_{S}$ has certain symmetry which does not show up (or is broken) at lower scales. If screening is realized as discussed in this paper, this symmetry propagates immediately to the light neutrino sector. $M_{S}$ can therefore be the origin of a specific "neutrino symmetry" which is not seen in the quark and in the charged lepton sectors. So, screening allows us to reconcile special "neutrino" symmetry [9] and the quark-lepton symmetry.

The examples considered in sec. 4.1)illustrate this possibility, since the matrices 42 15156) have certain symmetries. These symmetries are protected from large corrections in the supersymmetric case, while they can be strongly broken in the non-supersymmetric versions, or when some other interactions and fields beyond the SM or MSSM exist.

\subsection{Quark-lepton complementarity}

According to the quark-lepton complementarity relation [12, 13, 14, 15], the lepton mixing is given by maximal mixing minus the quark mixing. This may imply an existence of certain structure in the lepton sector which generates maximal (bi-maximal) mixing and the quarklepton symmetry in some form. In models with screening mechanism, the mass matrix $M_{S}$ which has no analogy in the quark sector, can be the origin of the bi-maximal mixing. Then the CKM type mixing follows from the charged lepton mass matrix which is related to the mass matrix of the down quarks, so that $U_{e}=U_{C K M}$. In the lowest order (without radiative corrections) we find from eq. (41)

$$
m_{\nu}^{f}=\left[\frac{\langle\phi\rangle}{\langle\sigma\rangle}\right]^{2} U_{e}^{T} M_{S} U_{e}=\left[\frac{\langle\phi\rangle}{\langle\sigma\rangle}\right]^{2} U_{C K M}^{T} U_{b m}^{*} M_{S}^{\operatorname{diag}} U_{b m}^{\dagger} U_{C K M}
$$

where $U_{b m} \equiv R_{23}^{m} \times R_{12}^{m}$ is the bi-maximal mixing matrix and $R_{i j}^{m}$ are the $\pi / 4$-rotations in the $(i j)$ planes. So, the leptonic mixing matrix equals $U_{P M N S}=U_{C K M}^{\dagger} U_{b m}$. This realizes the so called "neutrino scenario" which leads to deviations from the exact quark-lepton complementarity relation [14.

The bi-maximal mixing is produced by the mass matrix [36]

$$
M_{S}^{\text {bimax }}=\left(\begin{array}{ccc}
D-C & B & -B \\
\ldots & D & C \\
\ldots & \ldots & D
\end{array}\right),
$$


where $B, C, D$ are arbitrary parameters. As we have found in section 4.2 in the non-supersymmetric case, such a matrix may be unstable with respect to radiative corrections: the $d=5$ operator corrections can, in particular, suppress the maximal 2-3 mixing. This can be easily seen for vanishing $B$ and small $D$, when $M_{S}$ reduces to a triangular structure which is not stable under radiative corrections, as we have shown in sec. 4.2 .

\subsection{Singular $M_{S}$}

Let us consider the special case of singular $M_{S}$, $\operatorname{det} M_{S}=0$, which can be a consequence of certain symmetry in $S$ sector. Now one can not use immediately (5) and the whole double seesaw mass matrix should be considered. In what follows we will show that the tree-level mass matrix of light neutrinos is still proportional to $M_{S}$, that is, eq. (5D) will hold even if $M_{S}$ is singular. For this we will compare the light neutrino mass spectra in the lowest approximation found from the whole double seesaw matrix $\mathcal{M}$ and from the matrix $m_{\nu}$ after decoupling of the heavy degrees of freedom in eq. (5).

According to eq. (5) the condition $\operatorname{det} M_{S}=0$ implies (at least one) zero eigenvalue in the spectrum of usual LH neutrinos. The same follows from the complete matrix. Indeed,

$$
\operatorname{det} \mathcal{M}=-(\operatorname{det} Y)^{2} \operatorname{det} M_{S}=0,
$$

and hence, zero eigenvalue of $M_{S}$ leads to a massless eigenstate of $\mathcal{M}$. The non-zero eigenvalues of the matrix $m_{\nu}, \xi_{i}$, coincide with the eigenvalues of the full matrix $\mathcal{M}$ up to corrections of the order $\langle\phi\rangle /\langle\sigma\rangle$. This can be seen by inserting $\xi_{i}$ in the characteristic polynomial of the complete matrix $\chi_{\mathcal{M}}[\lambda]$. The result is of order $\mathcal{O}\left((\langle\phi\rangle /\langle\sigma\rangle)^{8}\right) \sim 0$. Hence, $\xi_{i}$ are to a very good approximation the eigenvalues of $\mathcal{M}$.

There are no other light states, because the expansion of the polynomial

$$
\chi_{\mathcal{M}}[\lambda] \prod_{i}\left(\lambda-\xi_{i}\right)^{-1}
$$

in eigenvalues of the order $\langle\phi\rangle$ does not yield any new solutions. All other eigenvalues are at least of the order $\mathcal{O}\left(\langle\sigma\rangle^{2} / M_{S}\right)$.

A peculiarity of the spectrum of $\mathcal{M}$ is the appearance of one heavy Dirac particle, if the eigenstate of $M_{S}$ with zero mass, $S$, couples to only one right-handed neutrino $N$. This Dirac particle is formed by $S$ and $N$.

The mass spectrum can be easily obtained if $M_{S}=\operatorname{diag}\left(M_{S 1}, M_{S 2}, 0\right)$ in the basis where $Y_{N}=\operatorname{diag}\left(y_{1}, y_{2}, y_{3}\right)$. Apart from one zero mass which corresponds mainly to $\nu_{3}$, and two super heavy eigenvalues $M_{S 1}$ and $M_{S 2}$ for two singlets $S$, we find

$$
m_{1}=M_{S 1} \frac{\langle\phi\rangle^{2}}{\langle\sigma\rangle^{2}}, \quad m_{2}=M_{S 2} \frac{\langle\phi\rangle^{2}}{\langle\sigma\rangle^{2}}, \quad M_{1}=-\frac{y_{1}^{2}\langle\sigma\rangle^{2}}{M_{S 1}}, \quad M_{2}=-\frac{y_{1}^{2}\langle\sigma\rangle^{2}}{M_{S 2}}, \quad M_{D S}=y_{3}\langle\sigma\rangle,
$$

that is, two light neutrinos predominantly given by $\nu_{1,2}$ with masses $m_{1}$ and $m_{2}$, two heavy neutrinos mostly consisting of $N_{1,2}$ with masses $M_{1}$ and $M_{2}$ and one heavy Dirac particle of the GUT scale mass $M_{D S}$ which is formed by $N_{3}$ and $S_{3}$. The light eigenstates are mainly composed of the left-handed neutrinos and the mixing with other neutral leptons is the order $\mathcal{O}(\langle\phi\rangle /\langle\sigma\rangle)$.

The coincidence of the spectrum of $m_{\nu}$ and the spectrum of light states of $\mathcal{M}$ is related essentially to the fact that the relation between $m_{\nu}$ and $M_{S}$ is linear, and the characteristic 
polynomial is linear in the eigenvalues for the non-degenerate case. The same conclusion holds for $M_{S}$ with two zero eigenvalues.

Let us consider the effect of radiative corrections for this singular case. As long as all contributions to a Majorana mass matrix $m_{\nu}$ receive the same quantum corrections, the RG evolution does not generate non-zero masses from vanishing masses [37. However, between the mass thresholds of the $\mathrm{RH}$ neutrinos, there are two contributions from the decoupling of the RH neutrinos which are renormalized differently. One contribution is due to the $\mathrm{d}=5$ operator decoupled and the other is due to the contribution of the $\mathrm{RH}$ neutrinos which are not decoupled yet $\left(Y_{\nu} M_{N}^{-1} Y_{\nu}^{T}\right)$ in the intervals $M_{2}-M_{3}$ and $M_{1}-M_{2}$. Hence, the generated mass is proportional to the additional renormalization factor $\delta Z_{\kappa}$ from the $\mathrm{d}=5$ operator between the thresholds and the mismatch between the two mass contributions, i.e. the deviation of the unitary matrix transforming from the eigenbasis of the $\mathrm{d}=5$ operator to the eigenbasis of $Y_{\nu} M^{-1} Y_{\nu}^{T}$ between the thresholds from the unit matrix (See sec. 4 in [31].). In the supersymmetric version, all contributions to the Majorana mass matrix receive the same quantum corrections, and hence zero mass eigenvalues remain zero.

\section{Screening, Grand Unification and flavor symmetry}

Let us discuss the possible origin of the screening structure which results from the mass matrix (44) together with the condition ([6). The texture of the matrix (44) with zero 1-1, 1-3, and 2-2 blocks can be obtained, e.g., assigning the lepton numbers

$$
L(\nu)=L(S)=1, \quad L(N)=-1, \quad L(\phi)=0, \quad L(\sigma)=0
$$

so that the lepton numbers of the blocks in the mass matrix (4) equal

$$
L(\mathcal{M})=\left(\begin{array}{ccc}
2 & 0 & 2 \\
0 & -2 & 0 \\
2 & 0 & 2
\end{array}\right) .
$$

Then the texture (4) appears if the lepton number is only broken by the Majorana mass terms of $S$. The lepton number can be broken explicitly or spontaneously by the VEV of the new scalar field $\rho$ which has the lepton number $L(\rho)=-2$ and couples with $S$ only: $S^{T} Y_{S} S \rho$. (The coupling of Majoron with the SM fields in negligible.) The interaction $\nu^{T} S \rho$ is forbidden by the gauge symmetry. The possible non-renormalized term

$$
\frac{1}{M_{P l}} L S \phi \rho
$$

gives negligible effects due to small $\operatorname{VEV}\langle\phi\rangle$. The coupling of $\rho$ with $\nu$ is also forbidden by the gauge symmetry. The term $N N \rho$ is absent in the supersymmetric version due to holomorphy.

In the non-supersymmetric version or if also the left superfield $\rho^{c}$ exists, an extended gauge symmetry can forbid the 2-2 entry. Indeed, in left-right symmetric models $N$ enters the doublet of $S U(2)_{R}$ and the 2-2 block has gauge charge $(1,3)$. The corresponding mass term appears once the Higgs triplet $\Delta_{R}$ exists.

The whole texture (41) can be a consequence of the gauge symmetry. Let us consider the $S U(2)_{L} \times S U(2)_{R} \times U(1)$ symmetry [1, 38. The $\left[S U(2)_{L}, S U(2)_{R}\right]$ gauge properties of the 
mass matrix elements are

$$
G(\mathcal{M})=\left(\begin{array}{ccc}
{[3,1]} & {[2,2]} & {[2,1]} \\
\ldots & {[1,3]} & {[1,2]} \\
\ldots & \ldots & {[1,1]}
\end{array}\right) .
$$

The required matrix structure is generated if the Higgs bi-doublet with the electroweak VEV, the RH doublet with GU-scale VEV and the singlet with $M_{P l}$ scale VEV exist. No particular lepton number prescription is needed.

In the context of $\mathrm{SO}(10)[\underline{3}, \nu$ and $N$ belong to the 16-plet and $S$ is a singlet. The required texture can be generated by the following Yukawa interactions:

$$
Y_{\nu} 16 \times 16 \times 10_{H}+Y_{N} \mathbf{1 6} \times \mathbf{1} \times \overline{\mathbf{1 6}}_{H}+Y_{S} \mathbf{1} \times \mathbf{1} \times \mathbf{1}_{H},
$$

where $\mathbf{1 0}_{H}, \overline{\mathbf{1 6}}_{H}$, and $\mathbf{1}_{H}$ are the Higgs multiplets. To generate matrix (4) $\mathbf{1 0}_{H}$ should acquire the electroweak VEV, $\overline{\mathbf{1 6}}_{H}$ - the GU scale VEV in $N$ (SU $U_{5}$ singlet) direction and $\mathbf{1}_{H}$ - the Planck scale VEV.

The interactions (69) do not produce quark mixing, and the Dirac masses of quarks and leptons are equal at the GUT scale. So, realistic model should contain some additional sources of the fermion masses which may, in general, destroy screening. For instance, one can introduce 126-plet of Higgses. Then VEVs of 126-plet in the "triplet" directions will generate the 1-1 and 2-2 blocks and therefore should be small enough. An alternative is the non-renormalizable interactions of the type $16 \times \mathbf{1 6} \times \mathbf{1 6}_{H} \times \mathbf{1 6}_{H} / M_{P l}$.

Apparently, the interactions (69) do not lead to relation (66). The equality or proportionality of the Yukawa couplings (6) can appear due to

- further unification of $\nu$ and $S$;

- certain flavor symmetry.

Let us comment on these two possibilities.

1). The neutral leptons $\nu, N$ and $S$, can be embedded into a single representation $\mathbf{2 7}$ of the gauge symmetry group $E_{6}$ [4. Notice that in this case there are two additional neutral leptons in each generation: $S^{\prime}$ and $S^{\prime \prime}$. The screening structure - the matrix (4) with the equality ([6) can be generated by the couplings

$$
Y_{27} \mathbf{2 7} \times \mathbf{2 7} \times \mathbf{2 7} \mathbf{7}_{H}+Y_{351_{S}} \mathbf{2 7} \times \mathbf{2 7} \times(\mathbf{3 5 1} \mathbf{S})_{H}+Y_{351_{A}} \mathbf{2 7} \times \mathbf{2 7} \times(\mathbf{3 5 1} \mathbf{A})_{H},
$$

where the 27-plet as well as the symmetric and antisymmetric 351-plets of Higgses are introduced.

In terms of the maximal subgroup $\mathrm{SU}(3)_{L} \times \mathrm{SU}(3)_{R} \times \mathrm{SU}(3)_{C} \subset E_{6}$, the leptons transform as $L \sim(\overline{\mathbf{3}}, \mathbf{3}, \mathbf{1})$. The $(\mathrm{SU}(3))^{3}$ assignment of the neutral leptons is

$$
\nu \sim L_{3}^{\dot{2}}, \quad N \sim L_{2}^{\dot{3}}, \quad S \sim L_{3}^{\dot{3}}, S^{\prime} \sim L_{1}^{\dot{1}}, S^{\prime \prime} \sim L_{2}^{\dot{2}} .
$$

The neutral components of Higgs multiplets $H, H_{A}$ and $H_{S}$ which can acquire VEVs belong to

$$
\begin{aligned}
& H \subset(\overline{\mathbf{3}}, \mathbf{3}, \mathbf{1}) \subset \mathbf{2 7}_{H} \\
& H_{S} \subset(\overline{\mathbf{3}}, \mathbf{3}, \mathbf{1})+(\mathbf{6}, \overline{\mathbf{6}}, \mathbf{1}) \subset\left(\mathbf{3 5} \mathbf{1}_{\mathbf{S}}\right)_{H} \\
& H_{A} \subset(\overline{\mathbf{3}}, \mathbf{3}, \mathbf{1})+(\overline{\mathbf{3}}, \overline{\mathbf{6}}, \mathbf{1})+(\mathbf{6}, \mathbf{3}, \mathbf{1}) \subset\left(\mathbf{3 5 1}_{\mathbf{A}}\right)_{H} .
\end{aligned}
$$


The Higgs multiplets $H$ and $H_{A}$ generate the Dirac structure ${ }^{5}$ and the Majorana mass terms are generated by $H_{S}[23$.

Notice that it is not possible to get a Dirac mass term of $S=L_{3}^{\dot{3}}$ with $N=L_{2}^{\dot{3}}$, using $\mathbf{2 7}_{H}$ Higgs multiplet due to antisymmetric (in SU(3) indices) interactions. The symmetric Higgs representation $(\mathbf{3 5 1} \mathbf{S})_{H}$ can generate all mass terms of neutral leptons required for screening. Indeed, the VEVs of $H_{\{\dot{2} \dot{3}\}}^{\{23\}}$ and $H_{\{\dot{2} \dot{3}\}}^{\{33\}}$ can be of order of the electroweak scale and of the $\mathrm{SU}(2)_{R}$ breaking scale correspondingly. Also its $H_{\{\dot{3} \dot{3}\}}^{\{33\}}$ component generates the Majorana mass of $S$. However, with a single $\left(\mathbf{3 5 1}_{\mathbf{S}}\right)_{H}$, the structure of matrix $M_{S}$ will be the same as the structure of the Dirac mass matrices. One can introduce a second $(\mathbf{3 5 1} \mathbf{S})_{H}$ which produces the mass matrix of $S$ of different structure.

Another more promising possibility is to use the antisymmetric $(\mathbf{3 5 1} \mathbf{A})_{H}$ Higgs multiplet which can generate all necessary Dirac matrices. It does not produce the Majorana masses of $S$ which can be done using $(\mathbf{3 5 1} \mathbf{S})_{H}$ so that the structure of $M_{S}$ is different from that of Dirac structures.

The following VEVs of the $(\mathbf{3 5 1} \mathbf{A})_{H}$ and $(\mathbf{3 5 1} \mathbf{S})_{H}$ components ${ }^{6}$

$$
\begin{aligned}
\left\langle\left(H_{A}\right)_{1}^{i}\right\rangle & \simeq \mathcal{O}\left(\mathrm{SU}(2)_{L} \text { breaking scale }\right) \\
\left\langle\left(H_{A}\right)_{1}^{\{33\}}\right\rangle & \simeq \mathcal{O}\left(\mathrm{SU}(2)_{R} \text { breaking scale }\right) \\
\left\langle\left(H_{S}\right)_{\{\dot{3} \dot{3}\}}^{\{33\}}\right\rangle \simeq\left\langle\left(H_{A}\right)_{3}^{\dot{3}}\right\rangle & \simeq \mathcal{O}\left(\mathrm{SU}(3)_{L} \times \mathrm{SU}(3)_{R} \text { breaking scale }\right)
\end{aligned}
$$

lead to the double seesaw. Indeed, in the basis $\left(\nu, N, S, S^{\prime}, S^{\prime \prime}\right)$ they generate mass matrix

$$
\left(\begin{array}{ccccc}
0 & -Y_{351_{A}}\left\langle\left(H_{A}\right)_{1}^{i}\right\rangle & 0 & 0 & 0 \\
\ldots & 0 & -Y_{351_{A}}\left\langle\left(H_{A}\right)_{1}^{\{33\}}\right\rangle & 0 & 0 \\
\ldots & \ldots & Y_{351_{S}}\left\langle\left(H_{S}\right)_{\{\dot{3} \dot{3}\}}^{\{33\}}\right\rangle & Y_{351_{A}}\left\langle\left(H_{A}\right)_{1}^{i}\right\rangle & 0 \\
\ldots & \ldots & \ldots & 0 & Y_{351_{A}}\left\langle\left(H_{A}\right)_{3}^{\dot{3}}\right\rangle \\
\ldots & \ldots & \ldots & \ldots & 0
\end{array}\right)
$$

with the required structure for $\nu, N$ and $S$. In addition to the mass spectrum of the double seesaw, there is one pseudo-Dirac particle formed by $S^{\prime}$ and $S^{\prime \prime}$ with a mass of the order of the $\mathrm{SU}(3)_{L} \times \mathrm{SU}(3)_{R}$ breaking scale.

Notice that interactions with $\mathbf{2} \mathbf{7}_{H}$ Higgs multiplet can be used to generate sub-leading effects, correcting the masses of quarks and producing some deviation from complete screening if needed. Furthermore, as the VEVs of components contributing to the 1-3 and 2-2 element break $\mathrm{SU}(2)_{L}$ invariance, they can only lead to entries of the order of the electroweak scale.

2). The equality of the Yukawa matrices (6) can be a consequence of certain flavor symmetry (See reviews [39, 40] and references therein.). It implies that the flavor charges of $\nu$ and $S$ are equal and the flavor symmetry uniquely determines the Yukawa couplings of these components with $N$. For this to happen, the flavor symmetry should be non-Abelian.

\footnotetext{
${ }^{5}$ The $\mathbf{2 7} \mathbf{7}_{H}$ and $(\mathbf{3 5 1} \mathbf{A})_{H}$ can not generate Majorana mass terms because the corresponding Yukawa interactions has to be antisymmetric in the $\mathrm{SU}(3)$ indices.

${ }^{6}$ The upper indices are $\mathrm{SU}(3)_{L}$ indices in the fundamental $(\mathbf{3})$ representation and the lower ones belong to $\mathrm{SU}(3)_{R}$. The 6 of SU(3) is represented by symmetric $3 \times 3$ matrices and described by 2 symmetrized indices. Dotted indices belong to the complex conjugate representation $(\overline{\mathbf{3}})$. Flavor indices are suppressed.
} 
In the Froggatt-Nielsen (F-N) approach 41] with U(1) flavor symmetry (and in $\mathrm{SO}(10)$ context) we can write

$$
\left(Z_{a b} \mathbf{1 6}_{a} \times \mathbf{1 6} \mathbf{6}_{b} \times \mathbf{1 0}_{H}+Z_{a b}^{\prime} \mathbf{1 6}_{a} \mathbf{1}_{b} \times \overline{\mathbf{1 6}}_{H}\right)\left(\frac{H_{F N}}{M_{F N}}\right)^{q_{a}+q_{b}},
$$

where $H_{F N}$ is the F-N scalar with $U(1)$ charge $-1, M_{f}$ is the scale where the operators (72) are formed and $q_{a}$ are the $U(1)$ charges of fermionic fields $\mathbf{1 6}_{a} . Z_{a b}$ and $Z_{a b}^{\prime}$ are supposed to be couplings of the order 1 , but for screening to work one needs to impose an additional proportionality condition $Z_{a b}=c Z_{a b}^{\prime}(c=$ const $)$.

Let us comment on a completely different possibility. If $S$ belongs to the 16-plet and $N$ is the singlet of $\mathrm{SO}(10)$, the required equality of the Yukawa couplings (6) is automatically reproduced. The screening structure can be generated by interactions

$$
Y 16 \times 1 \times \overline{\mathbf{1 6}}_{H}+Y_{S} \mathbf{1 6} \times \mathbf{1 6} \times \overline{\mathbf{1 2 6}}_{H}+Y_{q} \mathbf{1 6} \times \mathbf{1 6} \times 10_{H},
$$

if $\overline{\mathbf{1 6}}_{H}$ has the electroweak VEV in the " $\nu_{L}$ " direction and the GUT scale VEV in the " $\nu_{R}$ " direction, and $\overline{\mathbf{1 2 6}}_{H}$ has the Planck scale VEV in the direction of the SU(5) singlet. The last term in (73) gives the Dirac masses of quarks and leptons and also the Dirac mass term for $\nu$ and $S$. The mass matrix generated by (73) equals

$$
\mathcal{M}=\left(\begin{array}{ccc}
\sim 0 & Y\left\langle\overline{\mathbf{1 6}}_{H}\right\rangle & Y_{q}\left\langle\mathbf{1 0}_{H}\right\rangle \\
Y^{T}\left\langle\overline{\mathbf{1 6}}_{H}\right\rangle^{T} & \sim 0 & Y^{T}\left\langle\overline{\mathbf{1 6}}_{H}\right\rangle^{T} \\
Y_{q}\left\langle\mathbf{1 0}_{H}\right\rangle & Y\left\langle\overline{\mathbf{1 2 6}}_{H}\right\rangle & Y_{S}\left\langle\overline{\mathbf{1 2 6}}_{H}\right\rangle
\end{array}\right) .
$$

The 126-plet can also contribute to the 1-1 and 1-3 blocks. However, now $Y_{\nu}$ and $Y_{N}$ are not related to the Dirac matrices of quarks, and the problem of screening does not exist from the beginning. The usual seesaw contribution due to Dirac matrices which are related to the quark mass matrices $Y_{q}$ is suppressed by large (Planck) scale. That is, here we deal with suppression and not screening of the Dirac structures. Also in this case it would be more natural to identify the RH neutrino with $S$.

\section{Conclusions}

1). We studied screening of the Dirac flavor structure in the neutrino masses and mixings. Screening is realized in the context of double seesaw mechanism if the Dirac type Yukawa couplings of both seesaw steps are proportional to each other. The flavor structure of the light neutrino mass matrix is determined (in lowest order) by the flavor structure of matrix $M_{S}$ - the Majorana mass matrix of new singlets at the Planck scale, while the Dirac flavor structure is completely screened. The scale of the neutrino masses is correctly determined by the scales involved in the double seesaw as $M_{P l} v_{E W}^{2} / M_{G U}^{2}$.

2). The Planck-scale Majorana mass matrix $M_{S}$ violates lepton number, while at the same time it may have rich flavor symmetries. Screening translates these symmetries of $M_{S}$ directly into symmetries of $m_{\nu}$. The structure of $M_{S}$ can therefore be the origin of specific "neutrino symmetries" which do not show up in the quark sector. In particular, $M_{S}$ can be degenerate or quasi-degenerate leading (after the inclusion of radiative corrections) to a quasi-degenerate 
spectrum of light neutrinos. $M_{S}$, and consequently $m_{\nu}$, can also have certain symmetries which result in maximal 2-3 mixing and small 1-3 mixing. A very interesting possibility is that $M_{S}$ may have the bi-maximal structure which explains the quark-lepton complementarity. Screening allows therefore to reconcile the quark-lepton symmetry expressed as (approximate) equality of the Dirac mass matrices of quarks and leptons with the drastically different patterns of quark and lepton mixings. Thus the screening mechanism offers interesting possibilities in GUT model building.

3). The screening mechanism involves the cancellation of mass matrices which arise upon symmetry breaking at very different energy scales, namely the electroweak scale and the GUT scale. These matrices have different gauge properties leading to differences in the radiative corrections which can destroy screening. We studied stability of screening with respect to renormalization group effects.

It has been found that screening is stable in the MSSM and the external renormalization of the effective $d=5$ operator can be small. This means that the structure of the light neutrino mass matrix is still mostly determined by $M_{S}$, with small radiative corrections after renormalization group running is included.

In contrast, in the case of the $\mathrm{SM}$, due to the $\mathrm{d}=5$ operator corrections, the screening is unstable for certain structures of $M_{S}$. The form of the light neutrino mass matrix $m_{\nu}$ can differ strongly from that of $M_{S}$. This can, in fact, be used to explain some features of the light neutrinos via renormalization group effects.

4). Finally, the structure of the mass matrix (4) which leads to screening can be obtained using the lepton number or/and gauge symmetry. We outlined how screening could be realized in GUTs. The equality of the Dirac type Yukawa coupling matrices of $\nu$ and $N$ and of $S$ and $N$ can be a consequence of certain (horizontal) flavor symmetry or further unification of the fields. The latter can be realized, e.g., within the framework of $E_{6}$ gauge models.

\section{Acknowledgment}

The authors are grateful to B. Stech for valuable comments. The work of A.Yu.S. was supported by Alexander von Humboldt Foundation (the Humboldt Research Award). This work was also supported by the "Deutsche Forschungsgemeinschaft" in the "Sonderforschungsbereich 375-95 für Astro-Teilchenphysik" and under project number RO-2516/3-1.

\section{A Appendix}

We use GUT charge normalization for the hypercharge, i.e. $\frac{3}{5}\left(g_{1}^{\mathrm{GUT}}\right)^{2}=\left(g_{1}^{\mathrm{SM}}\right)^{2}$. The factors $Z$ describing the LL approximation are obtained from the counterterms in Ref. 30.

\section{A.1 SM}

In the SM extended by RH neutrinos, the wave function renormalization of the $\mathrm{RH}$ neutrinos is given by

$$
\stackrel{(n)}{Z}_{N}=\exp \left(\frac{1}{16 \pi^{2}} \stackrel{(n)}{Y}{ }_{\nu}^{\dagger} Y_{\nu} \ln \frac{M_{n}}{M_{n+1}}\right)
$$


and collecting the contributions from the renormalization of the left-handed doublets

$$
\beta_{Y_{\nu}}^{L}=\frac{1}{32 \pi^{2}}\left(\stackrel{(n)}{Y_{\nu} Y_{\nu}^{\dagger}}+Y_{e} Y_{e}^{\dagger}\right)
$$

the Higgs doublet

$$
\beta_{Y_{\nu}}^{\phi}=\frac{1}{32 \pi^{2}}\left(2 \operatorname{tr}\left(\stackrel{(n)}{Y_{\nu} Y_{\nu}^{\dagger}}+Y_{e} Y_{e}^{\dagger}+3 Y_{u} Y_{u}^{\dagger}+3 Y_{d} Y_{d}^{\dagger}\right)-\frac{9}{10} g_{1}^{2}-\frac{9}{2} g_{2}^{2}\right)
$$

and the vertex correction to $Y_{\nu}$

$$
\beta_{Y_{\nu}}^{Y_{\nu}}=-\frac{1}{8 \pi^{2}} Y_{e} Y_{e}^{\dagger}
$$

the external renormalization in the effective theory with $n \mathrm{RH}$ neutrinos yields

$$
\begin{array}{r}
\left.\stackrel{(n)}{Z_{\text {ext }}=\exp \left(\frac { 1 } { 3 2 \pi ^ { 2 } } \left(\stackrel{(n)}{Y_{\nu} Y_{\nu}^{\dagger}}-3 Y_{e} Y_{e}^{\dagger}+2 \operatorname{tr}\left(\stackrel{(n)}{Y_{\nu} Y_{\nu}^{\dagger}+(n)}+Y_{e} Y_{e}^{\dagger}\right.\right.\right.}+3 Y_{u} Y_{u}^{\dagger}+3 Y_{d} Y_{d}^{\dagger}\right) \\
\left.\left.-\frac{9}{10} g_{1}^{2}-\frac{9}{2} g_{2}^{2}\right) \ln \frac{M_{n}}{M_{n+1}}\right) .
\end{array}
$$

Neglecting the thresholds in the charged lepton sector and the quark sector, the expression for the external renormalization factor $Z_{\text {ext }}^{\mathrm{SM}}$ describing the total external renormalization can be further approximated to

$$
\begin{aligned}
Z_{\mathrm{ext}}^{\mathrm{SM}}=\exp & \left(\frac{1}{32 \pi^{2}} \sum_{n=0}^{3}\left[\begin{array}{l}
(n) \\
Y_{\nu} Y_{\nu}^{\dagger}+(n)
\end{array}+2 \operatorname{tr}\left(\begin{array}{l}
(n) \\
Y_{\nu} Y_{\nu}^{\dagger}
\end{array}\right)\right] \ln \frac{M_{n}}{M_{n+1}}\right. \\
& \left.+\frac{1}{32 \pi^{2}}\left[-3 Y_{e} Y_{e}^{\dagger}+2 \operatorname{tr}\left(Y_{e} Y_{e}^{\dagger}+3 Y_{u} Y_{u}^{\dagger}+3 Y_{d} Y_{d}^{\dagger}\right)-\frac{9}{10} g_{1}^{2}-\frac{9}{2} g_{2}^{2}\right] \ln \frac{\langle\phi\rangle}{\Lambda}\right)
\end{aligned}
$$

Here for uniformity of presentation we have denoted

$$
M_{0} \equiv\langle\phi\rangle, \quad M_{4} \equiv \Lambda
$$

The renormalization effect due to the additional vertex corrections to the $\mathrm{d}=5$ operator is given by

$$
\stackrel{(n)}{Z}{ }_{\kappa}=\exp \left(\frac{1}{16 \pi^{2}}\left(\lambda+\frac{9}{10} g_{1}^{2}+\frac{3}{2} g_{2}^{2}\right) \ln \frac{M_{n}}{M_{n+1}}\right) .
$$

The mass of the right-handed neutrinos receives only corrections from the wave function renormalization to arbitrary loop order. 


\section{A.2 MSSM}

In the MSSM extended by RH neutrinos, there are no vertex corrections due to the nonrenormalization theorem and the wave function renormalization yields

$$
\begin{aligned}
& \stackrel{(n)}{Z_{L}}=\exp \left(\frac{1}{32 \pi^{2}}\left(2 Y_{e} Y_{e}^{\dagger}+2 \stackrel{(n)}{Y_{\nu}} Y_{\nu}^{\dagger}-\frac{3}{5} g_{1}^{2}-3 g_{2}^{2}\right) \ln \frac{M_{n}}{M_{n+1}}\right) \\
& \stackrel{(n)}{Z_{N}}=\exp \left(\frac{1}{8 \pi^{2}} \stackrel{(n)}{Y}{ }_{\nu}^{\dagger} Y_{\nu} \ln \frac{M_{n}}{M_{n+1}}\right) \\
& \stackrel{(n)}{Z_{\phi}}=\exp \left(\frac{1}{32 \pi^{2}}\left(\operatorname{tr}\left(6 Y_{u} Y_{u}^{\dagger}+2 \stackrel{(n)}{Y_{\nu} Y_{\nu}^{\dagger}}\right)-\frac{3}{5} g_{1}^{2}-3 g_{2}^{2}\right) \ln \frac{M_{n}}{M_{n+1}}\right) \text {. }
\end{aligned}
$$

The external renormalization factor $Z_{\text {ext }}^{\mathrm{MSSM}}$ is given by the product of the wave function renormalization of the left-handed doublet with the Higgs doublet

$$
\stackrel{(n)}{Z_{\text {ext }}}=\stackrel{(n)}{Z_{L}} \stackrel{(n)}{\phi}_{\phi}
$$

because the two wave function renormalization factors commute. As the neutrino Yukawa couplings only change at the thresholds (up to 1 loop order), the external renormalization factor can be further approximated by

$$
\begin{aligned}
Z_{\mathrm{ext}}^{\mathrm{MSSM}}=\exp \left(\frac{1}{16 \pi^{2}} \sum_{n=0}^{3}\left(\stackrel{(n)}{Y_{\nu} Y_{\nu}^{\dagger}}+\operatorname{tr}\left(\stackrel{(n)}{Y_{\nu} Y_{\nu}^{\dagger}}\right)\right) \ln \frac{M_{n}}{M_{n+1}}\right. & \\
& \left.+\frac{1}{16 \pi^{2}}\left(Y_{e} Y_{e}^{\dagger}-\frac{3}{5} g_{1}^{2}-3 g_{2}^{2}+3 \operatorname{tr}\left(Y_{u} Y_{u}^{\dagger}\right)\right) \ln \frac{\langle\phi\rangle}{\Lambda}\right) .
\end{aligned}
$$




\section{References}

[1] J. C. Pati and A. Salam, Lepton number as the fourth color, Phys. Rev. D10 (1974), 275.

[2] H. Georgi and S. L. Glashow, Unity of all elementary particle forces, Phys. Rev. Lett. 32 (1974), 438.

[3] H. Georgi, The state of the art - gauge theories. (talk), AIP Conf. Proc. 23 (1975), 575;

H. Fritzsch and P. Minkowski, Unified interactions of leptons and hadrons, Ann. Phys. 93 (1975), 193.

[4] F. Gursey, P. Ramond, and P. Sikivie, A universal gauge theory model based on E6, Phys. Lett. B60 (1976), 177; Y. Achiman and B. Stech, Quark lepton symmetry and mass scales in an E6 unified gauge model, Phys. Lett. B77 (1978), 389; Q. Shafi, E(6) as a unifying gauge symmetry, Phys. Lett. B79 (1978), 301; R. Barbieri, D. V. Nanopoulos, and A. Masiero, Hierarchical fermion masses in E6, Phys. Lett. B104 (1981), 194.

[5] P. Minkowski, mu $\rightarrow$ e gamma at a rate of one out of 1-billion muon decays?, Phys. Lett. B67 (1977), 421.

[6] T. Yanagida, Horizontal gauge symmetry and masses of neutrinos, in Proceedings of the Workshop on The Unified Theory and the Baryon Number in the Universe (O. Sawada and A. Sugamoto, eds.), KEK, Tsukuba, Japan, 1979, p. 95; S. L. Glashow, The future of elementary particle physics, in Proceedings of the 1979 Cargèse Summer Institute on Quarks and Leptons (M. Lévy, J.-L. Basdevant, D. Speiser, J. Weyers, R. Gastmans, and M. Jacob, eds.), Plenum Press, New York, 1980, p. 687; M. Gell-Mann, P. Ramond, and R. Slansky, Complex spinors and unified theories, in Supergravity (P. van Nieuwenhuizen and D. Z. Freedman, eds.), North Holland, Amsterdam, 1979, p. 315; R. N. Mohapatra and G. Senjanović, Neutrino mass and spontaneous parity violation, Phys. Rev. Lett. 44 (1980), 912.

[7] Particle Data Group, S. Eidelman et al., Review of particle physics, Phys. Lett. B592 (2004), 1.

[8] A. Strumia and F. Vissani, Implications of neutrino data circa 2005, (2005), hep-ph/0503246

[9] T. Fukuyama and H. Nishiura, Mass matrix of Majorana neutrinos, (1997), hep-ph/9702253 E. Ma and M. Raidal, Neutrino mass, muon anomalous magnetic moment, and lepton flavor nonconservation, Phys. Rev. Lett. 87 (2001), 011802, hep-ph/0102255; C. S. Lam, A 2-3 symmetry in neutrino oscillations, Phys. Lett. B507 (2001), 214, hep-ph/0104116; K. R. S. Balaji, W. Grimus, and T. Schwetz, The solar LMA neutrino oscillation solution in the Zee model, Phys. Lett. B508 (2001), 301, hep-ph/0104035; P. F. Harrison and W. G. Scott, mu - tau reflection symmetry in lepton mixing and neutrino oscillations, Phys. Lett. B547 (2002), 219, hep-ph/0210197.

[10] H. V. Klapdor-Kleingrothaus, I. V. Krivosheina, A. Dietz, and O. Chkvorets, Search for neutrinoless double beta decay with enriched Ge-76 in Gran Sasso 1990-2003, Phys. Lett. B586 (2004), 198, hep-ph/0404088. 
[11] T. Kitabayashi and M. Yasue, S(2L) permutation symmetry for left-handed mu and tau families and neutrino oscillations in an $S U(3) L x U(1) N$ gauge model, Phys. Rev. D67 (2003), 015006, hep-ph/0209294; W. Grimus and L. Lavoura, A discrete symmetry group for maximal atmospheric neutrino mixing, Phys. Lett. B572 (2003), 189, hep-ph/0305046; Y. Koide, H. Nishiura, K. Matsuda, T. Kikuchi, and T. Fukuyama, Universal texture of quark and lepton mass matrices and a discrete symmetry Z(3), Phys. Rev. D66 (2002), 093006, hep-ph/0209333; Y. Koide, Universal texture of quark and lepton mass matrices with an extended flavor $2 i_{-}^{-} \dot{3}$ symmetry, Phys. Rev. D69 (2004), 093001, hep-ph/0312207; R. N. Mohapatra, theta(13) as a probe of mu i-i tau symmetry for leptons, JHEP 10 (2004), 027, hep-ph/0408187; W. Grimus et al., Non-vanishing U(e3) and $\cos (2$ theta(23)) from a broken Z(2) symmetry, Nucl. Phys. B713 (2005), 151, hep-ph/0408123; X. G. He and A. Zee, Some simple mixing and mass matrices for neutrinos, Phys. Lett. B560 (2003), 87, hep-ph/0301092.

[12] A. Y. Smirnov, (2004), Neutrinos: 'Annus mirabilis', hep-ph/0402264

[13] M. Raidal, Prediction Theta(c) + Theta(sol) $=$ pi/4 from flavor physics: A new evidence for grand unification?, Phys. Rev. Lett. 93 (2004), 161801, hep-ph/0404046.

[14] H. Minakata and A. Y. Smirnov, Neutrino mixing and quark lepton complementarity, Phys. Rev. D70 (2004), 073009, hep-ph/0405088.

[15] P. H. Frampton and R. N. Mohapatra, Possible gauge theoretic origin for quark-lepton complementarity, JHEP 01 (2005), 025, hep-ph/0407139; J. Ferrandis and S. Pakvasa, QLC relation and neutrino mass hierarchy, (2004), hep-ph/0412038, S. K. Kang, C. S. Kim, and J. Lee, Quark-lepton complementarity with renormalization effects through threshold corrections, (2005), hep-ph/0501029. A. Datta, L. Everett, and P. Ramond, Cabibbo haze in lepton mixing, (2005), hep-ph/0503222; S. Antusch, S. F. King, and R. N. Mohapatra, Quark lepton complementarity in unified theories, (2005), hep-ph/0504007.

[16] K. S. Babu and S. M. Barr, Large neutrino mixing angles in unified theories, Phys. Lett. B381 (1996), 202, hep-ph/9511446; S. M. Barr, Predictive models of large neutrino mixing angles, Phys. Rev. D55 (1997), 1659, hep-ph/9607419; C. H. Albright and S. M. Barr, Fermion masses in SO(10) with a single adjoint Higgs field, Phys. Rev. D58 (1998), 013002, hep-ph/9712488; G. Altarelli and F. Feruglio, Neutrino mass textures from oscillations with maximal mixing, Phys. Lett. B439 (1998), 112, hep-ph/9807353; G. Altarelli and F. Feruglio, Models of neutrino masses from oscillations with maximal mixing, JHEP 11 (1998), 021, hep-ph/9809596.

[17] A. Y. Smirnov, Seesaw enhancement of lepton mixing, Phys. Rev. D48 (1993), 3264, hep-ph/9304205.

[18] M. Tanimoto, Seesaw enhancement of neutrino mixing due to the right-handed phases, Phys. Lett. B345 (1995), 477, hep-ph/9503318; T. K. Kuo, G.-H. Wu, and S. W. Mansour, Mass hierarchies and the seesaw neutrino mixing, Phys. Rev. D61 (2000), 111301, hep-ph/9912366; G. Altarelli, F. Feruglio, and I. Masina, Large neutrino mixing from small quark and lepton mixings, Phys. Lett. B472 (2000), 382, hep-ph/9907532; S. Lavignac, I. Masina, and C. A. Savoy, Large solar angle and seesaw mechanism: A bottom-up perspective, Nucl. Phys. B633 (2002), 139, hep-ph/0202086; A. Datta, F.S. Ling, and P. Ramond, Correlated hierarchy, Dirac masses and large mixing angles, 
Nucl. Phys. B671 (2003), 383, hep-ph/0306002; M. Bando, S. Kaneko, M. Obara, and M. Tanimoto, Can symmetric texture reproduce neutrino bi-large mixing?, Phys. Lett. B580 (2004), 229, hep-ph/0309310.

[19] R. N. Mohapatra, Mechanism for understanding small neutrino mass in superstring theories, Phys. Rev. Lett. 56 (1986), 561; R. N. Mohapatra and J. W. F. Valle, Neutrino mass and baryon-number nonconservation in superstring models, Phys. Rev. D34 (1986), 1642.

[20] A. Y. Smirnov, Alternatives to the seesaw mechanism, (2004), hep-ph/0411194

[21] O. Vives, Understanding the differences in neutrino and charged fermion flavour structures, (2005), hep-ph/0504079.

[22] S. M. Barr, A different see-saw formula for neutrino masses, Phys. Rev. Lett. 92 (2004), 101601, hep-ph/0309152; C. H. Albright and S. M. Barr, Leptogenesis in the type III seesaw mechanism, Phys. Rev. D69 (2004), 073010, hep-ph/0312224.

[23] B. Stech and Z. Tavartkiladze, Fermion masses and coupling unification in E(6): Life in the desert, Phys. Rev. D70 (2004), 035002, hep-ph/0311161.

[24] B. Grzadkowski and M. Lindner, Non-linear evolution of Yukawa couplings, Phys. Lett. B193 (1987), 71.

[25] B. Grzadkowski, M. Lindner, and S. Theisen, Non-linear evolution of Yukawa couplings in the double Higgs and supersymmetric extensions of the Standard Model, Phys. Lett. B198 (1987), 64.

[26] J. A. Casas, J. R. Espinosa, A. Ibarra, and I. Navarro, Naturalness of nearly degenerate neutrinos, Nucl. Phys. B556 (1999), 3, hep-ph/9904395.

[27] J. A. Casas, J. R. Espinosa, A. Ibarra, and I. Navarro, Nearly degenerate neutrinos, supersymmetry and radiative corrections, Nucl. Phys. B569 (2000), 82, hep-ph/9905381.

[28] S. F. King and N. N. Singh, Renormalisation group analysis of single right-handed neutrino dominance, Nucl. Phys. B591 (2000), 3, hep-ph/0006229.

[29] S. F. King and N. N. Singh, Inverted hierarchy models of neutrino masses, Nucl. Phys. B596 (2001), 81, hep-ph/0007243.

[30] S. Antusch, J. Kersten, M. Lindner, and M. Ratz, Neutrino mass matrix running for non-degenerate see-saw scales, Phys. Lett. B538 (2002), 87, hep-ph/0203233.

[31] S. Antusch, J. Kersten, M. Lindner, M. Ratz, and M. A. Schmidt, Running neutrino mass parameters in see-saw scenarios, JHEP 03 (2005), 024, hep-ph/0501272.

[32] M. T. Grisaru, W. Siegel, and M. Roček, Improved methods for supergraphs, Nucl. Phys. B159 (1979), 429.

[33] N. Seiberg, Naturalness versus supersymmetric nonrenormalization theorems, Phys. Lett. B318 (1993), 469, hep-ph/9309335. 
[34] M. Fukugita and T. Yanagida, Baryogenesis without grand unification, Phys. Lett. 174B (1986), 45.

[35] S. Davidson and A. Ibarra, A lower bound on the right-handed neutrino mass from leptogenesis, Phys. Lett. B535 (2002), 25, hep-ph/0202239; W. Buchmuller, P. Di Bari, and M. Plumacher, Cosmic microwave background, matter-antimatter asymmetry and neutrino masses, Nucl. Phys. B643 (2002), 367, hep-ph/0205349 ; G. F. Giudice, A. Notari, M. Raidal, A. Riotto, and A. Strumia, Towards a complete theory of thermal leptogenesis in the SM and MSSM, Nucl. Phys. B685 (2004), 89, hep-ph/0310123.

[36] F. Vissani, (1997), A study of the scenario with nearly degenerate Majorana neutrinos, hep-ph/9708483 V. D. Barger, S. Pakvasa, T. J. Weiler, and K. Whisnant, Bi-maximal mixing of three neutrinos, Phys. Lett. B437 (1998), 107, hep-ph/9806387]; A. J. Baltz, A. S. Goldhaber, and M. Goldhaber, The solar neutrino puzzle: An oscillation solution with maximal neutrino mixing, Phys. Rev. Lett. 81 (1998), 5730, hep-ph/9806540; H. Georgi and S. L. Glashow, Neutrinos on earth and in the heavens, Phys. Rev. D61 (2000), 097301, hep-ph/9808293; I. Stancu and D. V. Ahluwalia, L/E-flatness of the electron-like event ratio in Super-Kamiokande and a degeneracy in neutrino masses, Phys. Lett. B460 (1999), 431, hep-ph/9903408.

[37] P. H. Chankowski and S. Pokorski, Quantum corrections to neutrino masses and mixing angles, Int. J. Mod. Phys. A17 (2002), 575, hep-ph/0110249.

[38] R. N. Mohapatra and J. C. Pati, A 'natural' left-right symmetry, Phys. Rev. D11 (1975), 2558; G. Senjanović and R. N. Mohapatra, Exact left-right symmetry and spontaneous violation of parity, Phys. Rev. D12 (1975), 1502.

[39] S. F. King, Neutrino mass models, Rept. Prog. Phys. 67 (2004), 107, hep-ph/0310204.

[40] G. Altarelli and F. Feruglio, Models of neutrino masses and mixings, (2004), hep-ph/0405048

[41] C. D. Froggatt and H. B. Nielsen, Hierarchy of quark masses, Cabibbo angles and CP violation, Nucl. Phys. B147 (1979), 277. 